\title{
Visualização no ensino de Ciências uma perspectiva para a integração de atividades experimentais
}

\author{
Visualization in Science education a perspective for the integration of experimental activities \\ Visualización en la enseñanza de las Ciencias una perspectiva para la integración de actividades
}

experimentales

Recebido: 14/01/2021 | Revisado: 18/01/2021 | Aceito: 21/01/2021 | Publicado: 25/01/2021

Helen Clemes Cardoso

ORCID: http://orcid.org/0000-0002-2271-6742 Universidade Federal de Santa Catarina, Brasil E-mail: helenclemes@gmail.com

Tatiana da Silva

ORCID: http://orcid.org/0000-0002-6677-6317 Universidade Federal de Santa Catarina, Brasil E-mail: tatiana.silva@ufsc.br

\begin{abstract}
Resumo
Ensinar ciências está diretamente associado a envolver os estudantes em atividades que os auxiliem a compreender modelos científicos abstratos, o que torna as experiências visuais fundamentais no processo de ensino-aprendizagem. Nessa perspectiva, a visualização no ensino de ciências apresenta uma abordagem teórica que busca idealizar os processos cognitivos, envolvidos em atividades que buscam favorecer essas experiências por meio dos objetos de visualização (como, por exemplo, gráficos, tabelas, diagramas esquemáticos). Entre estas experiências visuais podemos considerar as atividades experimentais, bastante difundidas no ensino de ciências, desenvolvidas no mundo concreto e/ou por meio das tecnologias digitais. Pesquisas recentes (por volta dos anos 2000) têm apresentado o contexto de integração de atividades experimentais reais (mundo concreto) e virtuais (tecnologias digitais), frente ao seu uso em configurações isoladas. Os resultados de aprendizagem comparativos têm evidenciado um cenário promissor com relação à integração dessas atividades. Deste modo o objetivo deste estudo foi analisar esses resultados com base na visualização, por meio dos objetos de visualização oferecidos nas atividades das pesquisas. Os resultados encontrados confirmaram nossa hipótese de que a integração se torna mais favorável ao ensino, pois oferece diferentes objetos de visualização que se tornam complementares. Cenário este que não acontece ao usarmos apenas uma modalidade de atividade experimental.
\end{abstract}

Palavras-chave: Visualização no ensino de ciências; Objetos de visualização; Integração de atividades experimentais; Ensino.

\begin{abstract}
Science teaching is directly associated with involving students in activities that help them to understand abstract scientific models, making visual experiences fundamental in the teaching-learning process. In this perspective, visualization in science education presents a theoretical approach that seeks to understand the cognitive processes involved in activities that seek to favor these experiences, through visualization objects (such as graphs, tables, schematic diagrams). Among these visual experiences, we can consider the experimental activities, widespread in science teaching, developed in the concrete world and/or through digital technologies. Recent research (around the 2000s) has presented the scenario of integration of real experimental activities (concrete world) and virtual activities (digital technologies), in view of its use in isolated configurations. The comparative learning results have shown a promising scenario regarding the integration of these activities. Thus, the objective of this research was to analyze these results based on the visualization, through the visualization objects offered in the research activities. The results found confirmed our hypothesis that integration becomes more favorable to teaching, as it offers different visualization objects that become complementary. This scenario does not happen when using only one modality of experimental activity.
\end{abstract}

Keywords: Visualization in science education; Visualization objects; Integration of experimental activities; Teaching.

\section{Resumen}

La enseñanza de la ciencia está directamente asociada a involucrar a los estudiantes en actividades que les ayuden a comprender modelos científicos abstractos, haciendo de las experiencias visuales fundamentales en el proceso de enseñanza-aprendizaje. En esta perspectiva, la visualización en la educación científica presenta un abordaje teórico que busca comprender los procesos cognitivos involucrados en actividades que buscan favorecer estas experiencias, a través de objetos de visualización (como gráficos, tablas, diagramas esquemáticos). Entre estas experiencias visuales, podemos considerar las actividades experimentales, muy extendidas en la enseñanza de las ciencias, desarrolladas en 
el mundo concreto y/o mediante tecnologías digitales. Investigaciones recientes (alrededor de la década de 2000) han presentado el escenario de integración de actividades experimentales reales (mundo concreto) y actividades virtuales (tecnologías digitales), en vista de su uso en configuraciones aisladas. Los resultados de aprendizaje comparativo han mostrado un escenario prometedor en cuanto a la integración de estas actividades. Así, el objetivo de esta investigación fue analizar estos resultados a partir de la visualización, a través de los objetos de visualización ofrecidos en las actividades de investigación. Los resultados encontrados confirmaron nuestra hipótesis de que la integración se vuelve más favorable a la enseñanza, ya que ofrece diferentes objetos de visualización que se complementan. Este escenario no ocurre cuando se usa solo una modalidad de actividad experimental.

Palabras clave: Visualización en la enseñanza de las ciencias; Objetos de visualización; Integración de actividades experimentales; Ensenãnza.

\section{Visualização no Ensino de Ciências}

As experiências visuais estão diretamente associadas ao ensino de ciências e se fazem presentes nas diferentes atividades propostas na área, que têm por objetivo auxiliar os estudantes na compreensão dos conceitos abstratos presentes nos modelos científicos. O conceito de visualização, vindo da psicologia cognitiva, trouxe para o ensino de ciências uma nova perspectiva para compreender os processos cognitivos envolvidos no ensino-aprendizagem que despontam das experiências visuais dos estudantes, estabelecendo relação entre o mundo concreto e o abstrato.

Dessa forma, as informações apresentadas em um mundo concreto, sob uma perspectiva cognitivista, precisam ser internalizadas e significadas, para o campo abstrato. Podemos definir, então, a existência de um domínio interno (cognição/psicológico) e o domínio externo (concreto/físico), logo “[...] aprender envolve o processamento da informação como um meio de interação entre esses dois domínios” (MngunI, 2014, p. 2). Essa interação se dá em três etapas fundamentais (como idealizado pelo autor): internalização, conceitualização e externalização, compondo assim os processos cognitivos da visualização.

O conceito da visualização pode ser compreendido através de três ramificações conceituais: os objetos de visualização; a visualização introspectiva e a visualização interpretativa (Phillips, Norris E Macnab, 2010). A Visualização Introspectiva “[...] É Uma Construção Imaginativa De Uma Possível Experiência Visual” (Phillips, Norris E Macnab, 2010, p. 26), enquanto a visualização interpretativa é definida como a significação dessa construção imaginativa após a interação com os objetos de visualização. Assim, é possível fazer uma aproximação conceitual, entendendo a visualização introspectiva como a internalização, e a visualização interpretativa como a etapa de conceitualização. Etapas pertencentes ao domínio interno (cognição/psicológico) que despontam do domínio externo (concreto/físico) no qual são realizadas as atividades de ensino. E é neste domínio que podemos compreender o conceito fundamental da visualização, os objetos de visualização (OV).

Os OV podem ser entendidos como objetos físicos construídos com o objetivo de representar um ou mais conceitos, elaborados para o processo de instrução, que “[...] são vistos e interpretados por uma pessoa para o propósito de compreender outra coisa que não o próprio objeto" (Phillips, Norris, Macnab, 2010, p. 26). Assim, podemos entender que gráficos, tabelas, diagramas esquemáticos, por exemplo, são objetos de visualização utilizados no processo de ensino-aprendizagem. O gráfico é construído com objetivo de explicar, por exemplo, o movimento de um carro, envolvendo o conceito de tempo, deslocamento, aceleração e velocidade. A figura 1 apresenta um panorama dos conceitos e aproximações teóricas aqui discutidas. 
Figura 1- Esquema sobre a relação entre a visualização e suas etapas cognitivas com base em Mnguni (2014) e Phillips, Norris e Macnab (2010).

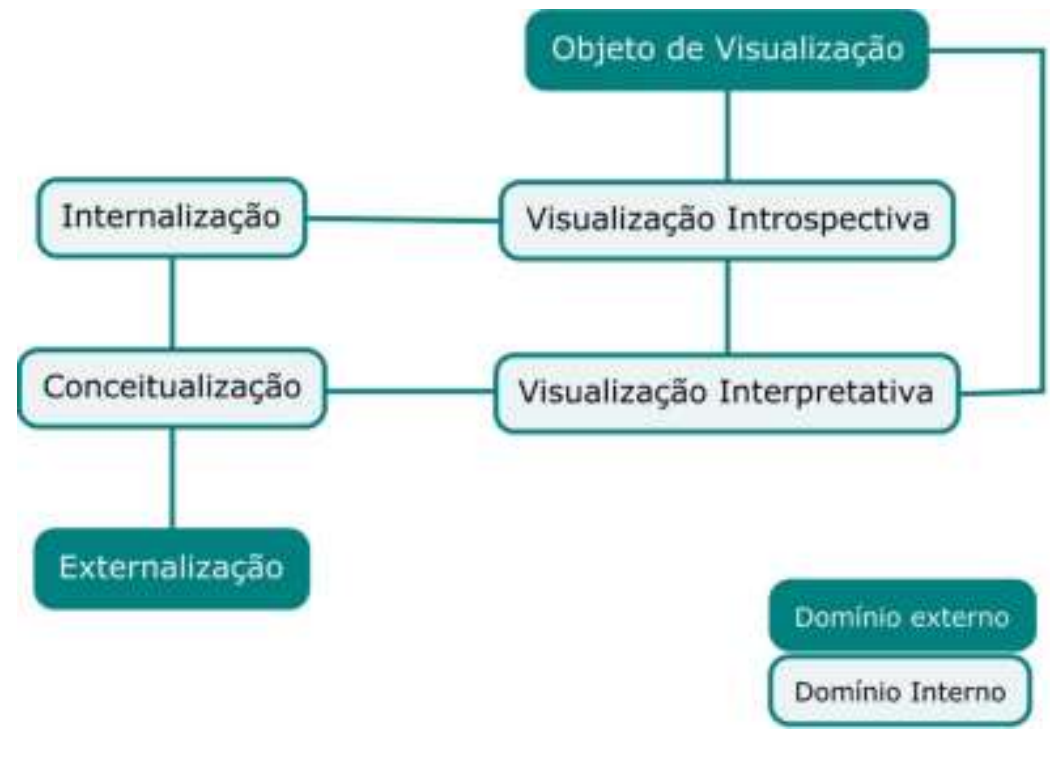

Fonte: Autoras.

Considerando os processos cognitivos teorizados pela visualização (Mnguni, 2014; Phillips, Norris, Macnab, 2010) podemos compreender que o processo de ensino desponta do domínio externo, no qual se fazem presentes os objetos de visualização. Assim, a elaboração e construção das atividades de ensino, que ocorrem neste domínio, serão responsáveis por auxiliar, ou não, os estudantes em suas internalizações e conceitualização (visualização introspectiva e interpretativa), por meio da exploração dos objetos de visualização. Esses objetos são responsáveis por oferecer experiências visuais que conduzam os estudantes na compreensão dos modelos científicos. Dessa forma, é fundamental compreender quais objetos de visualização participam do processo de ensino-aprendizagem e quais formatos existem, para que sejam selecionados de acordo com os objetivos de ensino traçados.

De acordo com Phillips e colaboradores (2010) é possível definir cerca de 12 objetos de visualização, sendo: i) diagramas esquemáticos (DE); ii) tabelas (T); iii) gráficos (G); iv) informação textual (IT); v) simbólico numérico (SN); vi) pictórico (P); vii) analogia (Ana); viii) realista (R); $\boldsymbol{i x}$ ) animação (Ani); $\boldsymbol{x}$ ) simulação (S); xi) interatividade (I); xii) domínio de validade $(D V)$. Cada um desses objetos de visualização deve ser utilizado quando necessário, para explanar os conceitos e modelos científicos abordados. Os i) diagramas esquemáticos (DE) por exemplo, podem ser usados com o objetivo de apresentar um ou mais conceitos por meio de uma simplificação imagética, para que o mesmo seja visto de uma forma mais objetiva e direta, em menor escala e de modo mais fundamental. Na física os esquemas de circuitos elétricos, no qual se tem a simbologia relacionada à resistência, ao capacitor, aos fios elétricos e demais objetos incorporados, auxiliam os estudantes a visualizar sistemas elétricos complexos, de uma forma mais descomplicada.

As ii) tabelas $(T)$ são objetos de visualização bastante utilizados no ensino e têm como objetivo também, simplificar e reunir informações. O seu grande diferencial está na organização, apresentando sempre os dados por meio de colunas e linhas, estabelecendo uma relação entre as informações que a compõem. Na biologia esse objeto se faz presente no ensino da taxonomia, por exemplo. Esses objetos podem ser explorados, por exemplo, de maneira a pedir que os estudantes completem as informações faltantes ou sistematizem os dados coletados. Os iii) gráficos $(G)$ também servem ao propósito dos objetos de visualização discutidos anteriormente. Eles reúnem informações de forma a relacioná-las por meio de eixos, cruzando dados, apresentando estatísticas, e em casos mais complexos, auxiliando na construção de objetos no espaço, como esferas, cones, 
triângulos, quando aplicados na geometria espacial (sub área da matemática). Em formatos mais simples podem relacionar grandezas, como por exemplo, na física para apresentar o deslocamento de uma partícula em função do tempo, podendo obter a partir dele a sua velocidade.

O objeto de visualização iv) informação textual (IT) é, provavelmente, o mais difundido e utilizado no ensino, a forma escrita apresenta conceitos e situações de um modo explicativo, contextualizando e exemplificando as informações, construindo explanações por meio de uma narrativa escrita. Os livros didáticos, por exemplo, presentes nas disciplinas de ciências, apresenta entre os objetos de visualização em sua estrutura, como maior frequência o objeto informação textual. Outro objeto bastante encontrado também nos livros didáticos, principalmente nas áreas de exatas, como física e matemática, é o v) simbólico numérico $(S N)$, utilizado para apresentar valores e quantificar informações.

Com relação às informações retratadas por meio da construção de imagens temos objeto de visualização vi) pictórico $(P)$ que pode estar associado ao modelo didático ou ainda a apenas um conceito. A área de ciências o utiliza em todas as suas áreas, na biologia podemos tomar como exemplo os desenhos de plantas, na física os objetos utilizados para descrever as partículas envolvidas no modelo científico estudado (bola, carro, sol, lua), na química os béqueres com soluções, na matemática as figuras geométricas. Nesse caso podem estar associados ainda outros dois objetos de visualização, como o vii) analogia (Ana) que tem como objetivo apresentar uma forma ou característica utilizada para retratar outra informação que não o seu próprio significado, como por exemplo, a utilização de elipses e círculos para caracterizar respectivamente a trajetória e os planetas no modelo didático do sistema solar. Ou ainda o objeto viii) realista $(R)$, que descreve o objeto de forma real $\mathrm{e}$ fidedigna, ou no caso de atividades experimentais a utilização do objeto em si. As atividades experimentais, sejam elas desenvolvidas por meio das tecnologias digitais exploram também os objetos de visualização, como o objeto ix) animação (Ani) que tem como objetivo a execução do movimento previsto pelo modelo científico abordado, assim como o objeto $\boldsymbol{x}$ ) simulação $(S)$. A diferença entre ambos os objetos está no grau de liberdade para interação, no caso da animação não existe a possibilidade de alterar parâmetros iniciais, enquanto a simulação oferece essa alternativa, apresentando um espaço para que os estudantes possam inserir/modificar dados e/ou fazer ajustes.

A xi) interatividade (I) também é entendida como um objeto de visualização, que pode ou não se fazer presente em diferentes níveis nas atividades de ensino, com maior ou menor grau de liberdade. O grau de liberdade está associado às funções e possibilidades que os estudantes possuem de interferir no modelo que está sendo abordado, atividades reais de laboratório oferecem um elevado grau de interatividade, considerando que sua execução depende diretamente da montagem de experimentos executada pelos estudantes, além da coleta de dados.

Um outro objeto de visualização relevante para o ensino é o xii) domínio de validade (DV), que tem como objetivo a exploração do campo de validade teórica do modelo científico estudado. É consenso na área da pesquisa em ensino de ciências que os modelos científicos possuem um campo de validade de previsões que podem ser feitas com base em seus conceitos, e em momentos do ensino, extrapolar esses limites pode ser importante para discussões relacionadas à própria criação da ciência. Nesses casos, testar o domínio da validade se torna uma escolha correta, para expor os estudantes a experiências visuais no qual os modelos 'falham'.

Considerando este cenário teórico no qual os objetos de visualização são componentes fundamentais das atividades de ensino, a visualização no ensino de ciências se mostra um norteador teórico para pautar as atividades de ensino, assim como resultados de pesquisas da área. Assim, considerando pesquisas recentes que buscam uma nova configuração de atividades experimentais, há aquelas que integram as realizadas por meio das tecnologias digitais (computador, tablet, smartphone) com as executadas em espaço concreto e real, - como laboratórios, saídas de campo, passeios em museu, entre outros, cujo objetivo é o de comparar resultados de aprendizagem entre os grupos de estudantes que utilizam apenas uma modalidade de atividade 
experimental, com o grupo de estudantes que realizaram ambas. Essas pesquisas evidenciaram que contextos em que os resultados apresentados pelo grupo de integração foram melhores, frente aos grupos do uso em contexto isolado.

No entanto, esses resultados não são discutidos sob uma perspectiva teórica de ensino, com o objetivo de lançar um olhar sobre as atividades desenvolvidas. Tendo em vista que as atividades experimentais partem fundamentalmente de experiências visuais dos estudantes frente a objetos ou sistemas de objetos, podemos lançar um olhar para elas a partir da concepção da visualização, mais especificamente, dos objetos de visualização. Assim, a presente pesquisa teve como objetivo analisar as atividades realizadas nessas publicações, considerando os objetos de visualização presentes nas mesmas. Acreditase que há uma relação direta entre os resultados positivos à integração, pois por meio das duas atividades experimentais é possível abordar diferentes objetos de visualização, que se complementam de forma oferecer melhores experiências visuais de ensino favorecendo a promoção da visualização interpretativa. Entretanto, primeiramente é preciso conhecer o panorama dessas pesquisas que realizam a integração de atividades experimentais.

\section{O Panorama da Integração das Atividades Experimentais}

O presente estudo se caracteriza como pesquisa bibliográfica qualitativa. Para construir o corpo de pesquisas (bibliografia analisada) e construir o cenário dos estudos de integração de atividades experimentais foram realizados dois momentos de revisão, o primeiro no ano de 2015/02 ${ }^{1}$ e o segundo momento no ano de 2020/01. A busca por estas pesquisas foi realizada seguindo a metodologia de busca nas bases de dados através do conjuntos de palavras-chaves, no cenário nacional e internacional (pesquisas publicadas em inglês), de acordo com a Quadro 1.

\footnotetext{
${ }^{1}$ Este manuscrito é resultado de uma pesquisa de mestrado apresentada ao Programa de Pós-Graduação de Educação Científica e Tecnológica no ano de 2017, intitulada "Um olhar para a integração de atividades experimentais reais e virtuais segundo a visualização no ensino de ciências". O segundo momento de revisão foi realizado com a finalidade de encontrar eventuais pesquisas publicadas após o ano da defesa.
} 
Quadro 1- Relação de palavras-chave e base de dados utilizadas na revisão bibliográfica.

\begin{tabular}{|c|c|c|c|}
\hline \multicolumn{2}{|c|}{ BASE DE DADOS } & \multicolumn{2}{|c|}{ PALAVRAS-CHAVE } \\
\hline & & 2015 & 2020 \\
\hline $\begin{array}{l}\mathbf{N} \\
\mathbf{A} \\
\mathbf{C} \\
\mathbf{I} \\
\mathbf{O} \\
\mathbf{N} \\
\mathbf{A} \\
\mathbf{L}\end{array}$ & $\begin{array}{c}\text { Portal CAPES } \\
\text { Google } \\
\text { Acadêmico }\end{array}$ & $\begin{array}{c}\text { "Tecnologia" + "experimentação" + } \\
\text { "ensino de ciências" } \\
\text { "Tecnologia digital" + } \\
\text { "experimentação" + "ensino de } \\
\text { ciências" } \\
\text { "Experimentação" + "simulação" + } \\
\text { "ensino de ciências" } \\
\text { "Experimentação" + "modelagem" + } \\
\text { "ensino de ciências" }\end{array}$ & $\begin{array}{c}\text { "Experimentação real" + } \\
\text { "experimentação virtual" + "ensino } \\
\text { de ciências" } \\
\text { "Experimentação real" + "simulação } \\
\text { computacional"+ "ensino de } \\
\text { ciências" } \\
\text { "Experimentação real"+ } \\
\text { "modelagem computacional" + } \\
\text { "ensino de ciências" } \\
\text { "Laboratório real" + "laboratório } \\
\text { virtual" + "ensino de ciências" }\end{array}$ \\
\hline $\begin{array}{l}\mathbf{I} \\
\mathbf{N} \\
\mathbf{T} \\
\mathbf{E} \\
\mathbf{R} \\
\mathbf{N} \\
\mathbf{A} \\
\mathbf{C} \\
\mathbf{I} \\
\mathbf{O} \\
\mathbf{N} \\
\mathbf{A} \\
\mathbf{L}\end{array}$ & $\begin{array}{c}\text { Eric } \\
\text { Science Direct } \\
\text { Google } \\
\text { Acadêmico }\end{array}$ & $\begin{array}{l}\text { "Real experimentation" + simulation" } \\
\text { + "science teaching" } \\
\text { "Real experimentation" + modeling" + } \\
\text { "science teaching" } \\
\text { "Real experimentation" + technology" } \\
\text { + "science teaching" }\end{array}$ & $\begin{array}{c}\text { "Real experimentation" + "virtual } \\
\text { experimentation" + "science } \\
\text { teaching" } \\
\text { "Real experimentation" + } \\
\text { "computational modeling" + } \\
\text { "science teaching" } \\
\text { "Real experimentation" + } \\
\text { "computational simulation" + } \\
\text { "science teaching" } \\
\text { "Hand-on" + "virtual } \\
\text { experimentation" + "science } \\
\text { teaching" }\end{array}$ \\
\hline
\end{tabular}

Fonte: Autoras.

O primeiro momento de busca, realizado no segundo semestre de 2015, resultou em um total de 24 pesquisas, e o segundo momento de levantamento bibliográfico, que ocorreu no primeiro semestre de 2020, resultou em um total de 27 estudos. Neste cenário estão inclusos artigos, dissertações, teses, e monografias, publicados no cenário nacional e internacional (publicações em inglês). A figura 2 apresenta as pesquisas publicadas no período de 2001 a 2020, recorte temporal encontrado partindo das datas de realização/publicação dos estudos. De acordo com as pesquisas encontradas pode-se dizer que esses questionamentos relacionados a integração de atividades experimentais começaram a ser estudados por volta do início dos anos 2000, e de acordo com a Figura 2, houve um salto entre os anos de 2011 a 2015. 
Figura 2- Número de pesquisas publicadas no período de 2001 a 2020.

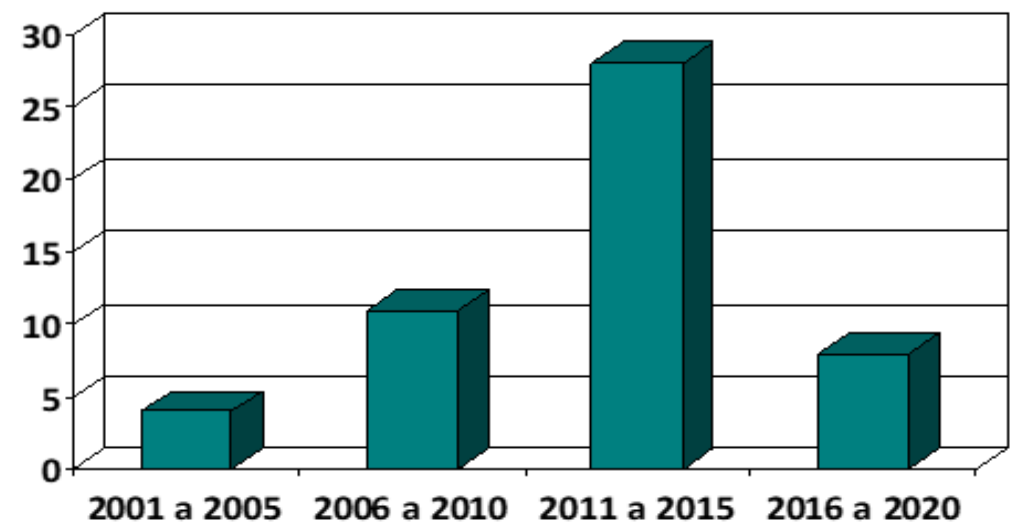

Fonte: Autoras.

Todos os estudos encontrados apresentavam a configuração de integração de atividades experimentais, em alguns casos, essa sequência foi analisada frente a alguma outra forma de ensino, iremos tratar aqui como grupo AER+AEV o caso em que a atividade experimental real (AER) foi aplicada junto a atividade experimental virtual (AEV). No caso das demais configurações que foram apresentadas nos estudos assumiremos: AER aplicação somente de atividade experimental real; AEV aplicação apenas da atividade experimental virtual; e no caso de haver um grupo com uso de metodologia tradicional, como afirmado pelos autores, GC configurando o grupo de controle.

Após a leitura na íntegra das pesquisas foram definidas seis categorias de acordo com os grupos de aplicação que configuraram os estudos, sendo: i) apenas AER+AEV; ii) AER+AEV versus AER; iii) AER+AEV versus GC; iv) AER versus $\mathrm{AEV}$; v) comparação entre os grupos AER, AEV e GC; vi) comparação entre os grupos AER, AEV, AER+AEV e GC. A Figura 3 apresenta as configurações de pesquisas encontradas e quantas pesquisas foram encontradas em cada categoria nas buscas realizadas no ano de 2015 e 2020.

Figura 3- Categorização da revisão bibliográfica.

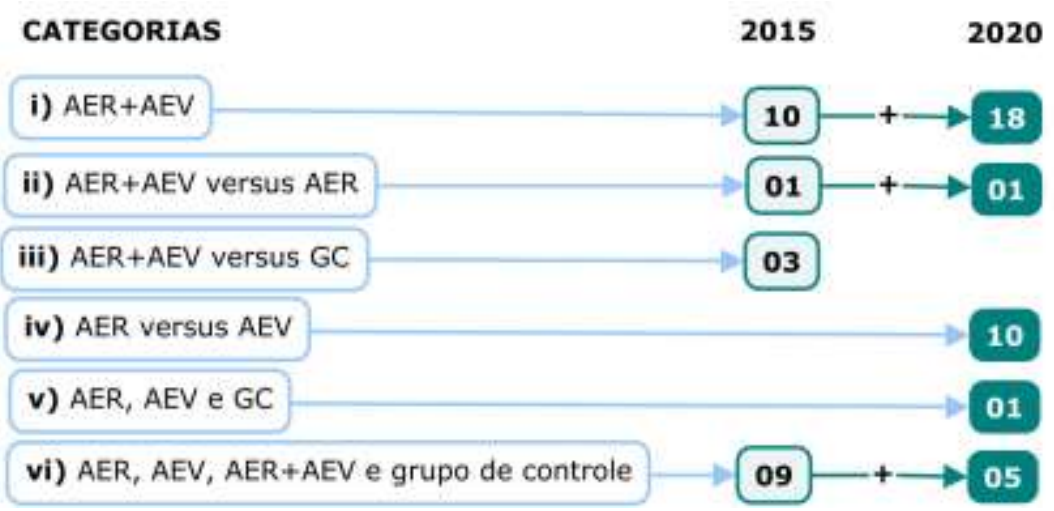

Fonte: Autoras.

Considerando que a categoria vi) apresenta todas as configurações de comparação entre os grupos de estudo, nossa atenção se volta para estas publicações. Para caracterizar estas pesquisas, aspectos de análise foram definidos a priori, com o intuito de mostrar o desenho desses estudos, sendo: Resultados, com relação à comparação entre os grupos do estudo; Tema 
abordado; Nível de ensino no qual a pesquisa foi aplicada; Ferramenta Virtual utilizada; Número de grupos; e total de Participantes envolvidos.

As informações analisadas estão apresentadas no Quadro 2, e são utilizadas as seguintes letras para representar: Nível de ensino: Fundamental (F), Ensino Médio (M), Graduação (G), Pós-Graduação (P). Para Tema, na Física: Eletromagnetismo (E), Gravitação (G), Termodinâmica (Te), Mecânica (M), na Biologia: Taxonomia (Ta). Com relação aos resultados comparativos entre os grupos dos estudos, lembrando que AER se refere às Atividades Experimentais Reais e AEV às Atividades Experimentais Virtuais: INTG - a integração apresenta melhores resultados que AEV ou AER utilizadas em contextos separados; V - AEV apresenta bons resultados com ou sem a utilização integrada de AER; EQ - AEV apresenta os mesmos resultados que AER quando utilizadas separadamente, ou seja, sem integração das atividades; IND - todos os grupos de atividades (AER+AEV, AEV ou AER) obtiveram os mesmos resultados.

Quadro 2- Caracterização das pesquisas da categoria vi).

\begin{tabular}{|c|c|c|c|c|c|c|c|}
\hline \multirow{2}{*}{\multicolumn{2}{|c|}{ Publicação }} & \multirow{3}{*}{$\begin{array}{c}\text { Resultado } \\
\text { INTG }\end{array}$} & \multirow{3}{*}{$\begin{array}{c}\text { Ferramenta } \\
\text { Virtual } \\
\text { Vitual Labs } \\
\text { Electricity }\end{array}$} & \multirow{3}{*}{$\begin{array}{c}\text { Tema } \\
\mathrm{E}\end{array}$} & \multirow{3}{*}{$\begin{array}{c}\begin{array}{c}\text { Nível de } \\
\text { Ensino }\end{array} \\
\text { F }\end{array}$} & \multicolumn{2}{|c|}{ Número de } \\
\hline & & & & & & \multirow{2}{*}{$\begin{array}{c}\text { Grupos } \\
3\end{array}$} & \multirow{2}{*}{$\begin{array}{c}\text { Participantes } \\
55\end{array}$} \\
\hline $\mathbf{A}$ & $\begin{array}{c}\text { Zacharia e } \\
\text { colaboradores, } \\
2014\end{array}$ & & & & & & \\
\hline B & $\begin{array}{l}\text { Zacharia e } \\
\text { Olympiou, } \\
2011\end{array}$ & IND & ThermoLab & $\mathrm{Te}$ & G & 5 & 234 \\
\hline C & $\begin{array}{l}\text { Sarabando, } \\
\text { Cavino e } \\
\text { Soares, } 2014\end{array}$ & INTG, V & Modellus & G & $\mathrm{F}$ & 3 & 142 \\
\hline D & $\begin{array}{c}\text { Trundle e Bell, } \\
2010\end{array}$ & IND & Starry Nigth & G & $\mathrm{P}$ & 3 & 157 \\
\hline $\mathbf{E}$ & $\begin{array}{l}\text { Farrokhnia e } \\
\text { Esmaipour, } \\
2010\end{array}$ & INTG, EQ & $\begin{array}{c}\text { Circuit } \\
\text { Construction } \\
\text { Kit }(\mathrm{PhET})\end{array}$ & $\mathrm{E}$ & G & 3 & 100 \\
\hline $\mathbf{F}$ & $\begin{array}{c}\text { Ünlü e Dökme, } \\
2011\end{array}$ & INTG & $\begin{array}{c}\text { Electricity } \\
\text { Simulation } \\
\text { Tools }\end{array}$ & $\mathrm{E}$ & $\mathrm{F}$ & 3 & 66 \\
\hline $\mathbf{G}$ & Mendes, 2009 & INTG & Modellus & M & M & 4 & 86 \\
\hline H & $\begin{array}{c}\text { Darrah e } \\
\text { colaboradores, } \\
2014\end{array}$ & IND & $\begin{array}{c}\text { Virtual Physics } \\
\text { Lab }\end{array}$ & M & G & 3 & 224 \\
\hline I & $\begin{array}{c}\text { Olympiou e } \\
\text { Zacharia, } 2011\end{array}$ & INT, EQ & Optilab & $\mathrm{O}$ & G & 3 & 70 \\
\hline $\mathbf{J}$ & $\begin{array}{l}\text { Zacharia e } \\
\text { Jong, } 2015\end{array}$ & & $\begin{array}{l}\text { Virtual Labs } \\
\text { Electricity }\end{array}$ & $\mathrm{E}$ & G & 5 & 194 \\
\hline $\mathbf{L}$ & $\begin{array}{l}\text { Pfeiffer, Scheter } \\
\text { e Gemballa, } \\
2012\end{array}$ & IND, V & Vídeos & $\mathrm{Ta}$ & G & 3 & 195 \\
\hline $\mathbf{M}$ & Otten, 2016 & IND & $\begin{array}{c}\text { Energy Skate } \\
\text { Park }\end{array}$ & M & M & 4 & 127 \\
\hline
\end{tabular}

Fonte: Autoras. 
As pesquisas de A à G, destacadas em cinza, foram as encontradas na revisão de 2015/02, e as destacadas em azul (H, I, J, L, M), foram encontradas da busca realizada em 2020/01. É interessante ressaltar que entre as doze pesquisas encontradas, seis apresentaram resultados de aprendizagem melhores no grupo que passou por ambas as atividades experimentais, ou seja, que integraram AER e AEV. Os resultados de aprendizagem foram analisados estaticamente pelos pesquisadores, buscando diferenças significativas através da comparação dos pós-testes entre os grupos. Entretanto, a razão pela qual essas diferenças são existentes fica em aberto nas pesquisas, apesar de alguns apontamentos terem sido feitos, não há uma discussão teóricametodológica sobre os aspectos presentes nas atividades que podem ter influenciado nos resultados.

É, nesse cenário, que entendemos que a visualização pode ser usada para explicar os resultados. Considerando que diferentes objetos de visualização se fazem presentes nas atividades de ensino, e estes estão diretamente associados ao ensinoaprendizagem, podemos entender que os resultados dessas pesquisas estão relacionados aos objetos de visualização explorados nas atividades experimentais aplicadas. Assim, na próxima seç̧ão são apresentados os objetos de visualização presentes nas atividades e a relação entre os resultados encontrados, com o objetivo de evidenciar nossa hipótese de que os resultados positivos de aprendizagem dos grupos que usaram a integração das atividades experimentais, está associado ao fato de que elas se complementam explorando grupos diferentes de objetos de visualização. A integração de atividades oferece aos estudantes experiências visuais mais completas que os auxiliam a construir soluções corretas, pois possuem diferentes formas de representar os conceitos nas soluções de problemas, o que lhes confere diferentes estratégias possíveis de resolução.

\section{Visualização, o Prisma para as Atividades Experimentais Reais e Virtuais}

Para construção da metodologia de análise da presente pesquisa, foi utilizado como base o autor Phillips e colaboradores (2010), em específico os conceitos de objetos de visualização descritos pelos pesquisadores, como parâmetro de análise das atividades desenvolvidas nas bibliográficas encontradas. Assim, todas as publicações encontradas foram lidas na íntegra, com a finalidade de compreender a proposta didática e o desenvolvimento de sua aplicação. As atividades experimentais foram analisadas sob a perspectiva das definições de objetos de visualização de acordo com a visualização no ensino de ciências.

O Quadro 3 apresenta as publicações em ordem alfabética, de acordo com a classificação apresentada anteriormente (Quadro 2), evidenciando os objetos presentes e ausentes de acordo com a configuração dos grupos. Para sinalizar a presença dos objetos de visualização foi utilizado a simbologia (s), no caso de ausência (.), em algumas situações não foi possível reconhecer se houve ou não a existência de determinados OV, assim não foi feito o preenchimento dos espaços. Lembrando que os objetos de visualização são: i) diagramas esquemáticos (DE); ii) tabelas (T); iii) gráficos (G); iv) informação textual $(I T)$; v) simbólico numérico (SN); vi) pictórico (P); vii) analogia (Ana); viii) realista $(R)$; ix) animação (Ani); x) simulação (S); xi) interatividade (I); xii) domínio de validade (DV). Com relação aos resultados: INTG - a integração apresenta melhores resultados que AEV ou AER utilizadas em contextos separados; V - AEV apresenta bons resultados com ou sem a utilização integrada de AER; EQ - AEV apresenta os mesmos resultados que AER quando utilizadas separadamente, ou seja, sem integração das atividades; IND - todos os grupos de atividades (AER+AEV, AEV ou AER) obtiveram os mesmos resultados. 
Quadro 3- Relação entre resultados comparativos e objetos de visualização presentes nas atividades experimentais desenvolvidas nas pesquisas.

\begin{tabular}{|c|c|c|c|c|c|c|c|c|c|c|c|c|c|c|}
\hline \multirow[b]{2}{*}{$\begin{array}{l}\mathbf{P} \\
\mathbf{U} \\
\mathbf{B} \\
\mathbf{L} \\
\mathbf{I} \\
\mathbf{C} \\
\mathbf{A} \\
\mathbf{C} \\
\tilde{\mathbf{A}} \\
\mathbf{O}\end{array}$} & \multirow[b]{2}{*}{$\begin{array}{c}\mathbf{R} \\
\mathbf{E} \\
\mathbf{S} \\
\mathbf{U} \\
\mathbf{L} \\
\mathbf{T} \\
\mathbf{A} \\
\mathbf{D} \\
\mathbf{O} \\
\mathbf{S}\end{array}$} & \multirow[b]{2}{*}{$\begin{array}{c}\mathbf{A} \\
\mathbf{T} \\
\mathbf{I} \\
\mathbf{V} \\
\mathbf{I} \\
\mathbf{D} \\
\mathbf{A} \\
\mathbf{D} \\
\mathbf{E}\end{array}$} & \multicolumn{12}{|c|}{ Objetos de Visualização } \\
\hline & & & $D E$ & $T$ & $G$ & $I T$ & $S N$ & $P$ & Ana & $R$ & $A n i$ & $S$ & $I$ & $D V$ \\
\hline \multirow[t]{3}{*}{$\mathbf{A}$} & \multirow[t]{3}{*}{ INTG } & AER & \multirow[t]{2}{*}{ s } & \multirow[t]{2}{*}{. } & \multirow[t]{2}{*}{. } & \multirow[t]{2}{*}{$\mathrm{s}$} & \multirow{2}{*}{ s } & \multirow{2}{*}{$\begin{array}{l}\mathrm{s} \\
\mathrm{s}\end{array}$} & \multirow[b]{2}{*}{$\mathrm{s}$} & \multirow[t]{2}{*}{. } & \multirow{2}{*}{. } & \multirow{2}{*}{$\begin{array}{l}\mathrm{S} \\
\mathrm{s}\end{array}$} & \multirow{2}{*}{$\begin{array}{l}\mathrm{s} \\
\mathrm{s}\end{array}$} & \multirow{2}{*}{. } \\
\hline & & $\mathrm{AEV}$ & & & & & & & & & & & & \\
\hline & & $\begin{array}{l}\mathrm{AER+} \\
\mathrm{AEV}\end{array}$ & $\mathrm{s}$ & . & . & s & s & s & $\mathrm{s}$ & . & . & $\mathrm{s}$ & $\mathrm{s}$ & . \\
\hline \multirow[t]{4}{*}{ B } & \multirow[t]{4}{*}{ IND } & AER & $\mathrm{s}$ & $\mathrm{s}$ & . & s & s & . & . & . & . & $\mathrm{s}$ & $\mathrm{s}$ & . \\
\hline & & $\mathrm{AEV}$ & & . & . & . & $\mathrm{s}$ & $\mathrm{s}$ & $\mathrm{s}$ & . & . & $\mathrm{s}$ & $\mathrm{s}$ & . \\
\hline & & $\begin{array}{l}\mathrm{AER+} \\
\mathrm{AEV}\end{array}$ & $\mathrm{s}$ & $\mathrm{S}$ & . & s & $\mathrm{s}$ & $\mathrm{s}$ & $\mathrm{S}$ & . & . & $\mathrm{s}$ & $\mathrm{s}$ & . \\
\hline & & $\begin{array}{l}\mathrm{AEV+} \\
\mathrm{AER}\end{array}$ & $\mathrm{s}$ & $\mathrm{S}$ & . & . & s & $\mathrm{s}$ & $\mathrm{S}$ & . & . & $\mathrm{s}$ & $\mathrm{s}$ & . \\
\hline \multirow[t]{3}{*}{$\mathbf{C}$} & \multirow{3}{*}{$\begin{array}{l}\text { INTG e } \\
\text { V }\end{array}$} & AER & & & & & & & . & . & & $\mathrm{s}$ & $\mathrm{S}$ & . \\
\hline & & $\mathrm{AEV}$ & & $\mathrm{S}$ & & & $\mathrm{s}$ & $\mathrm{s}$ & & $\mathrm{s}$ & & $\mathrm{s}$ & $\mathrm{S}$ & $\mathrm{s}$ \\
\hline & & $\begin{array}{l}\mathrm{AER+} \\
\mathrm{AEV}\end{array}$ & & $\mathrm{S}$ & & & $\mathrm{s}$ & $\mathrm{s}$ & & $\mathrm{s}$ & & $\mathrm{s}$ & $\mathrm{S}$ & $\mathrm{s}$ \\
\hline \multirow[t]{3}{*}{ D } & \multirow[t]{3}{*}{ IND } & AER & . & $\mathrm{S}$ & . & $\mathrm{s}$ & $\mathrm{s}$ & $\mathrm{s}$ & . & . & . & $\mathrm{S}$ & $\mathrm{S}$ & . \\
\hline & & $\mathrm{AEV}$ &. & $\mathrm{S}$ & . & $\mathrm{s}$ & $\mathrm{s}$ & $\mathrm{s}$ & . & . & . & $\mathrm{s}$ & $\mathrm{S}$ & . \\
\hline & & $\begin{array}{c}\mathrm{AER}+\mathrm{AE} \\
\mathrm{V}\end{array}$ & . & $\mathrm{S}$ & . & $\mathrm{S}$ & $\mathrm{s}$ & $\mathrm{s}$ & . & . & . & $\mathrm{S}$ & $\mathrm{S}$ & . \\
\hline \multirow[t]{3}{*}{$\mathbf{E}$} & \multirow{3}{*}{$\begin{array}{l}\text { INTG e } \\
\text { EQ }\end{array}$} & AER & & & & $\mathrm{s}$ & & & . & . & & $\mathrm{S}$ & $\mathrm{S}$ & . \\
\hline & & $\mathrm{AEV}$ & . & . & . & s & s & $\mathrm{s}$ & $\mathrm{s}$ & . & $\mathrm{s}$ & $\mathrm{S}$ & $\mathrm{S}$ & . \\
\hline & & $\begin{array}{l}\text { AER+ } \\
\text { AEV }\end{array}$ & & & & $\mathrm{S}$ & $\mathrm{s}$ & $\mathrm{S}$ & $\mathrm{s}$ & . & $\mathrm{s}$ & $\mathrm{s}$ & $\mathrm{S}$ & . \\
\hline \multirow[t]{3}{*}{$\mathbf{F}$} & \multirow[t]{3}{*}{ INTG } & AER & & & & & $\mathrm{s}$ & & . & . & & $\mathrm{S}$ & $\mathrm{S}$ & . \\
\hline & & AEV & $\mathrm{s}$ & . & . & $\mathrm{s}$ & $\mathrm{s}$ & . & $\mathrm{s}$ & . & . & $\mathrm{S}$ & $\mathrm{S}$ & . \\
\hline & & $\begin{array}{l}\mathrm{AER}+ \\
\mathrm{AEV}\end{array}$ & $\mathrm{s}$ & & & s & $\mathrm{s}$ & & $\mathrm{S}$ & . & & $\mathrm{S}$ & $\mathrm{S}$ & . \\
\hline $\mathbf{G}$ & INTG & AER & & & & & $\mathrm{s}$ & & . & . & $\mathrm{s}$ & $\mathrm{S}$ & $\mathrm{S}$ & $\mathrm{S}$ \\
\hline & & AEV & . & . & . & $\mathrm{s}$ & $\mathrm{s}$ & . & . & $\mathrm{s}$ & . & $\mathrm{s}$ & $\mathrm{s}$ & $\mathrm{s}$ \\
\hline & & $\begin{array}{l}\mathrm{AER}+ \\
\mathrm{AEV}\end{array}$ & & & & $\mathrm{S}$ & $\mathrm{s}$ & & & $\mathrm{S}$ & $\mathrm{S}$ & $\mathrm{S}$ & $\mathrm{S}$ & $\mathrm{S}$ \\
\hline $\mathbf{H}$ & IND & AER & & & & & & & & & $\mathrm{s}$ & & $\mathrm{S}$ & \\
\hline & & $\mathrm{AEV}$ & & $\mathrm{s}$ & & $\mathrm{s}$ & $\mathrm{s}$ & $\mathrm{s}$ & $\mathrm{S}$ & & . & $\mathrm{s}$ & $\mathrm{S}$ & \\
\hline & & $\begin{array}{l}\mathrm{AER}+ \\
\mathrm{AEV}\end{array}$ & & S & & $\mathrm{s}$ & $\mathrm{s}$ & $S$ & $\mathrm{~s}$ & & $\mathrm{~s}$ & $\mathrm{~s}$ & $\mathrm{~S}$ & \\
\hline
\end{tabular}




\begin{tabular}{|c|c|c|c|c|c|c|c|c|c|c|c|c|c|c|}
\hline \multirow{3}{*}{ I } & \multirow{3}{*}{$\begin{array}{l}\text { INTG e } \\
\text { EQ }\end{array}$} & AER & $\mathrm{S}$ & . & $\cdot$ & $\mathrm{s}$ & $\mathrm{s}$ & $\mathrm{S}$ & $\mathrm{S}$ & $\mathrm{s}$ & $\cdot$ & . & $\mathrm{S}$ & \\
\hline & & AEV & $\mathrm{s}$ & . & . & . & $\mathrm{s}$ & $\mathrm{s}$ & & . & . & & $\mathrm{s}$ & \\
\hline & & $\begin{array}{c}\mathrm{AER}+ \\
\mathrm{AEV}\end{array}$ & $\mathrm{s}$ & . & . & $\mathrm{s}$ & $\mathrm{s}$ & $\mathrm{s}$ & $\mathrm{s}$ & $\mathrm{s}$ & . & & $\mathrm{S}$ & \\
\hline \multirow{3}{*}{$\mathbf{J}$} & \multirow[t]{3}{*}{ IND } & AER & $\mathrm{s}$ & . & . & $\mathrm{s}$ & . & $\mathrm{s}$ & . & . & . & $\mathrm{S}$ & $\mathrm{S}$ & . \\
\hline & & AEV & . & . & . & . & $\mathrm{s}$ & $\mathrm{s}$ & $\mathrm{s}$ & . & . & $\mathrm{S}$ & $\mathrm{s}$ & . \\
\hline & & $\begin{array}{c}\text { AER+ } \\
\text { AEV }\end{array}$ & $\mathrm{s}$ & . & . & $\mathrm{s}$ & $\mathrm{s}$ & $\mathrm{s}$ & $\mathrm{s}$ & . & . & $\mathrm{S}$ & $\mathrm{S}$ & . \\
\hline \multirow{3}{*}{$\mathbf{L}$} & \multirow{3}{*}{$\begin{array}{l}\text { IND e } \\
\mathrm{V}\end{array}$} & AER & . & . & . & $\mathrm{s}$ & . & . & $\mathrm{s}$ & . & $\mathrm{s}$ & . & . & . \\
\hline & & AEV & . & . & . & . & . & . & $\mathrm{S}$ & . & $\mathrm{s}$ & . & . & . \\
\hline & & $\begin{array}{c}\text { AER+ } \\
\text { AEV }\end{array}$ & . & . & . & $\mathrm{S}$ & . & . & $\mathrm{s}$ & . & $\mathrm{S}$ & . & . & \\
\hline \multirow{3}{*}{$\mathbf{M}$} & \multirow[t]{3}{*}{ IND } & AER & . & $\mathrm{s}$ & . & $\mathrm{s}$ & . & . & . & $\mathrm{s}$ & . & $\mathrm{s}$ & $\mathrm{S}$ & \\
\hline & & AEV & . & $\mathrm{s}$ & & $\mathrm{s}$ & . & $\mathrm{s}$ & . & . & $\mathrm{s}$ & $\mathrm{s}$ & $\mathrm{s}$ & \\
\hline & & $\begin{array}{c}\mathrm{AER}+ \\
\mathrm{AEV}\end{array}$ & . & $\mathrm{s}$ & & $\mathrm{S}$ & . & $\mathrm{S}$ & . & $\mathrm{s}$ & $\mathrm{S}$ & $\mathrm{S}$ & $\mathrm{S}$ & \\
\hline
\end{tabular}

Fonte: Autoras.

Para maior confiabilidade para as discussões, vamos comparar o caso das publicações que obtiveram resultados diferentes, e no qual foi possível identificar a ausência ou presença de todos os objetos de visualização em todos os casos. A publicação A destacada em verde, apresentou o grupo que passou pela integração (INTG) com melhores resultados de aprendizagem, enquanto os estudos D e L destacados em amarelo, obtiveram resultados de aprendizagem (IND) indiferente entre os grupos. Se direcionarmos o olhar para os objetos de visualização explorados nas atividades desenvolvidas, no caso da pesquisa D e L, os mesmos objetos de visualização se fizeram presentes nas atividades experimentais reais e virtuais. A publicação D tinha como desenvolvimento das atividades a observação das fases da Lua a olho nu (AER) e por meio do software Starry Night. A observação feita pelos estudantes tinha de ser o registrado em uma tabela na qual tinham de anotar hora e dia e desenhar a área não iluminada da Lua. No caso da publicação L os estudantes de graduação tinham que identificar 15 espécies de peixes de água doce da Europa. As atividades desenvolvidas foram por meio de vídeo (AEV) e identificação dicotômica (AER), que se dá por meio de uma lista de características descritas. Todos os participantes do estudo foram, ao final das atividades experimentais, a uma visita ao aquário público da Alemanha.

No entanto, para a publicação A as atividades exploraram diferentes objetos de visualização, o que ofereceu aos estudantes diferentes experiências visuais com relação ao conteúdo abordado. O estudo abordou o conteúdo de eletromagnetismo, discutindo os conceitos de tensão, corrente e resistores, presentes na Lei de Ohm, explorando as atividades experimentais com base no livro Physics by Inquiry (Mcdermott, 1996).

$\mathrm{Na}$ atividade experimental real (AER) os estudantes utilizaram materiais (baterias idênticas, fios, interruptores e lâmpadas) para construírem os circuitos elétricos com configurações de uma e duas lâmpadas em duas configurações distintas (em paralelo e em série). Os estudantes do grupo experimental virtual (AEV) tiveram de realizar as mesmas configurações utilizando a ferramenta Virtual Labs Electrecity. O objetivo era fazer com que os estudantes, em ambas as atividades, observassem o brilho apresentado pela lâmpada nas diferentes configurações de circuito.

O grande diferencial entre os estudos D, L e A, é que os estudantes tiveram experiências visuais quase iguais no caso das publicações D e L, explorando os mesmos objetos de visualização. Diferente da publicação A, que ofereceu aos estudantes que realizaram as atividades virtuais e reais, experiências visuais distintas, pois cada atividade ofereceu uma gama diferente de 
OV. Em cada atividade o olhar dos estudantes foi direcionado para o que era importante naquele momento, no caso da AER o brilho da lâmpada foi o foco, e a montagem do circuito, o que ficou mais fácil para ser feito na AEV, que agora também apresentou a possibilidade de se visualizar os valores da tensão e da corrente elétrica. Nesse caso a atividade experimental real ofereceu um preparo para o desenvolvimento da AEV, e esta mostrou de forma mais clara e direta a relação entre a alteração da configuração do circuito e os valores referentes à tensão e corrente (Figura 4, destaque no retângulo em amarelo), o que era fundamental para a compreensão do tema.

Figura 4 - Imagem estática da atividade experimental virtual Virtual Labs Eletricity.

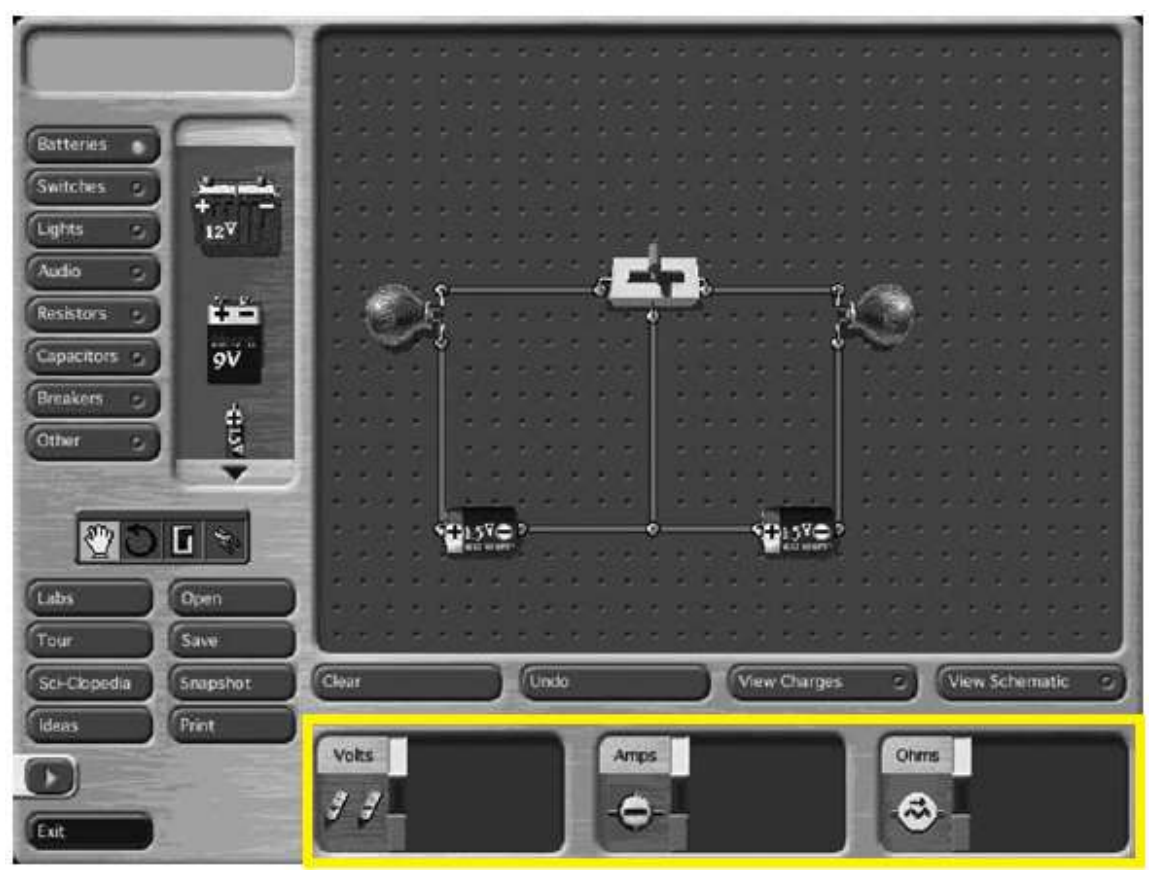

Fonte: Adaptada de Zacharia et al. (2014).

O principal nesta comparação entre os estudos é mostrar que as experiências visuais são influenciadas pelos objetos de visualização, e quando essas experiências são parecidas, direcionando o olhar dos estudantes para os mesmos pontos, ou seja, os mesmos objetos, os resultados de aprendizagem não se mostram positivos como nos casos em que o oposto acontece, quando integramos atividades com objetos distintos resultando em experiências diferentes.

Assim pode-se entender que a integração de atividades experimentais (reais e virtuais) é favorável quando ambas se beneficiam de distintos objetos de visualização, proporcionando ao estudante transitar entre os mesmos de forma complementar, ou seja, um ou mais objetos de cada atividade se integram e oferecem experiências visuais diferentes. De acordo com a teorização do processo cognitivo da visualização (Mnguni, 2014) a aprendizagem ocorre durante as etapas de internalização, conceitualização e externalização.

A internalização desponta dos objetos de visualização que são apresentados aos estudantes no domínio externo. Quantos mais objetos forem explorados nas atividades de ensino, diferentes serão as internalizações (visualizações introspectivas) que passando para o processo de conceitualização (visualização interpretativa), poderão ser usadas como estratégias para externalização dos conceitos pelos estudantes em resoluções de atividades.

No entanto, a quantidade de objetos não garante que os objetivos de ensino serão alcançados, é necessário que a intenção de uso dos mesmos seja clara para o professor, para que saiba como conduzir a atividade, para os estudantes, para que saibam o que devem observar, assim como estar alinhado aos objetivos de ensino. É preciso pensar na relevância do objeto de 
visualização utilizado de acordo com o modelo científico subjacente.

\section{Considerações Finais}

A percepção é um processo inerente ao ser humano, inconscientemente visualizamos e significamos objetos em nossa cognição, estas construções ocorrem de modo individual, mesmo que tenhamos a mesmas experiências visuais. Assim, podemos entender que visualizar (ver e significar) é a etapa inicial e essencial do ensino de um modelo científico, e compreender como esse processo se dá na cognição é fundamental para que se crie estratégias de ensino que alcancem os objetivos traçados. Os objetos de visualização pensados e construídos para a instrução devem ser elaborados e ensinados intencionalmente. As tabelas, gráficos, entre outros, não são claros por si só, precisam ser explorados com base em um planejamento e com um objetivo de ensino. Sobre quantos mais objetos os estudantes aprenderem e souberem compreender, significar e externalizar, melhores poderão ser os resultados de aprendizagem, pois mais ferramentas eles possuirão para construção do seu conhecimento. As análises feitas sobre os resultados das pesquisas e das atividades desenvolvidas sinalizam para validar a teorização da visualização.

É fundamental salientar que os objetos podem estar presentes em diferentes configurações de atividades. Entretanto, a atividade experimental, seja na modalidade virtual ou real, desempenha papel importante no ensino de ciências há algum tempo, seja de modo lúdico e demonstrativo, ou com o intuito de envolver os estudantes durante as atividades, ou ainda com o objetivo de oferecer uma aula diferenciada. Porém, partindo das análises construídas aqui, é possível ir além e dar um passo à frente no ensino com base na visualização, pois a mesma direciona em um caminho no qual pode-se pensar e construir essas atividades com maior clareza de suas implicações quanto ao ensino. Ou seja, a clareza de que a partir dessas atividades pode-se explorar os objetos de visualização e enriquecer as experiências visuais dos estudantes em contextos no qual a integração real e virtual ocorre.

Contudo, partindo do cenário aqui apresentado, segmentos de estudos podem ser desenvolvidos e elaborados, os objetos de visualização podem ser ampliados, suas definições podem ser aprofundadas, outras pesquisas de integrações podem ser feitas para ampliar os estudos a serem analisados. É possível também, construir um cenário de pesquisa de sequência didática aplicando a AER e AEV e sua integração, no qual as atividades sejam propostas e elaboradas com base nos objetos de visualização utilizados, buscando na externalização dos estudantes diferenças de aprendizagem entre grupos com experiências visuais distintas ou ainda analisando os objetos de visualização que os estudantes utilizam antes e depois das atividades, com o intuito de perceber se há uma evolução nas externalizações construídas.

\section{Agradecimentos}

Agradecemos a Fundação de Amparo à Pesquisa e Inovação do Estado de Santa Catarina (FAPESC), pelo fomento à presente pesquisa.

\section{Referências}

Darrah, M., Humbert, R., Finstein, J., Simon, M., \& Hopkins, J. (2014). Are virtual labs as effective as hands-on labs for undergraduate physics? A comparative study at two major universities. J Sci Educ Technol, 23,803-814.

Farrokhnia, M. R., \& Esmailpour, A (2010). A study on the impact of real, virtual and comprehensive experimenting on students' conceptual understanding of DC electric circuits and their skills in undergraduate electricity laboratory. Procedia Social and Behavioral Sciences. 2, 5474-5482.

McDermott, L. (1996). Physics by inquiry: an introduction to physics and the physical sciences, vol. 2: John Wiley \& Sons.

Mendes, J. F. (2009). O uso do software Modellus na integração entre conhecimentos teóricos e atividades experimentais de tópicos de Mecânica sob a perspectiva da aprendizagem significativa. Dissertação mestrado, Universidade de Brasília, Distrito Federal, DF, Brasil.

Mnguni, L. E. (2014). The theoretical cognitive process of visualization for science education. SpringerPlus. 34(184), 02-09. 
Research, Society and Development, v. 10, n. 1, e49510111981, 2021

(CC BY 4.0) | ISSN 2525-3409 | DOI: http://dx.doi.org/10.33448/rsd-v10i1.11981

Olympiou, G., \& Zazharia, Z. C. (2011). Blending physical and virtual manipulatives: an effort to improve students' conceptual understanding through science laboratory experimentation. Science Education, 96(1), 21-47.

Pfeiffer, V. D. I., Scheiter, K., \& Gemballa, S. (2012). Comparing and combining traditional teaching approaches and the use of video clips for learning how to identify species in an aquarium. Journal of Biological Education, 46(3), 140-148.

Phillips, L. M., Norris, S. P., \& Macnab, J. N. (2010). Visualization in Mathematics, reading and science education: Springer.

Sarbando, C., Cavino, J. P. \& Soares, A. A (2014). Contribuiton of a computer simulation to stundents learning of the physics concepts of weight and mass. Procedia technology, 13,112-121.

Trundle, K. C., \& Bell, R. L (2010). The use of a computer simulation to promove conceptual change: a quasi-experimental study. Computer and education, $54,1078-1088$

Ünlü Z. K., \& Dökme, I. (2011). The effect of three different teaching tools in science education on the students' attitudes towards computer. Procedia Social and Behavioral Sciences, 15, 2652-2657.

Zacharia, Z. C., \& Olympiou, G. (2011). Physical versus virtual manipulative experimentation in physics learning. Learning and Instruction, $21,317-331$.

Zacharia, Z. C., \& Jong, T. (2014). The effects on students' conceptual understanding of electric circuits of introducing virtual manipulatives within a Physical manipulatives-oriented curriculum. Cognition and Instruction, 32(2), 101-158.

Zacharia, Z. C., Michael, M., Olympiou, G., \& Papasozomenou, V. (2014). Blending physical and virtual manipulatives to improve primary school students' learning in physics. 2013, In C. P. Constantinou, N. Papadouris., \& A. Hadjigeorgiou (Eds.), E-Book Proceedings of the ES-ERA 2013 Conference: Science Education Research For Evidence-based Teaching and Co-herence in Learning. Part 16: Science in the primary school (co-ed. Editors of the strand chap-ter), (pp.2883-2989). Nicosia, Cyprus: European Science Education Research Association. ISBN: 978-9963-700-77-6 